
\title{
28 Research Soure \\ Moving the Goal Posts in movement pattern modification using a robotic force field
}

\author{
Yangmengfei Xu \\ University of Melbourne https://orcid.org/0000-0003-4320-0064 \\ Vincent Crocher ( $\nabla$ vincent.crocher@unimelb.edu.au ) \\ https://orcid.org/0000-0001-9643-8389 \\ Justin Fong \\ University of Melbourne \\ Ying Tan \\ University of Melbourne \\ Denny Oetomo \\ University of Melbourne
}

\section{Research}

Keywords: movement pattern modification, neurorehabilitation, robotic-assisted, synergy, motor control, motor learning, upper limb

Posted Date: May 6th, 2020

DOI: https://doi.org/10.21203/rs.3.rs-26382/v1

License: (c) (1) This work is licensed under a Creative Commons Attribution 4.0 International License.

Read Full License 


\title{
RESEARCH
}

\section{Moving the Goal Posts in movement pattern modification using a robotic force field}

\author{
Yangmengfei $\mathrm{Xu}^{*}$, Vincent Crocher, Justin Fong, Ying Tan and Denny Oetomo
}

\author{
${ }^{*}$ Correspondence: \\ y.xu100@student.unimelb.edu.au \\ UoM \& Fourier Robotics \\ Laboratory, Department of \\ Mechanical Engineering, The \\ University of Melbourne, Grattan \\ Street, 3010 Parkville, Australia \\ Full list of author information is \\ available at the end of the article
}

\begin{abstract}
Background: Moving the Goal Posts (MGP) typically refers to a progressive change of goal or objective - a classic mechanism to promote motor function improvement in neuro-rehabilitative therapy. However, this MGP idea is still missing in the literature of robotic-assisted strategies to improve quality of movement, which is of high-importance in motor neuro-rehabilitation. This study aims to investigate if an MGP approach in a robotic-assisted upper limb movement pattern modification leads to a larger swivel angle change on healthy subjects during a reaching task training.
\end{abstract}

Methodology: Randomised control single blind study. Healthy naive subjects were allocated into two groups: Control $(\mathrm{N}=10)$ and MGP $(\mathrm{N}=10)$ and performed the same reaching tasks under different interventions provided by a manipulandum robotic device. During the intervention, the robotic device applied an Indirect Shaping Control (ISC): a resistive viscous force applied at the subject's hand as a function of the subject's swivel angle to encourage a modification of the later towards a $10^{\circ}$ increase. In the MGP group, the goal was altered progressively towards the overall $10^{\circ}$ increase in swivel angle, while in the Control group, the goal was set constant to the $10^{\circ}$ from the beginning.

Results: A significant increase of the swivel angle, of $4.0^{\circ}$ and $6.2^{\circ}$ respectively, was observed in both groups $(p<0.05)$. A significantly larger change was observed in the MGP group $(p<0.001)$. All but one subject adapted into a new movement pattern, but no significant retention was observed in any of the two groups after intervention.

Conclusion: The superior effect of the intervention with the MGP approach compared to the constant goal demonstrates that the progressivity of learning plays an important role in this context and can ultimately lead to larger changes. Nevertheless, in both cases the effect of the intervention was small and longer interventions and/or steeper progressivity should be investigated to obtain larger changes. Retention was not observed in any group, but this may be different in a motor neuro-rehabilitation context where the newly taught movement pattern may result in additional movement capabilities or other benefits for neurologically impaired individuals.

Keywords: movement pattern modification; neurorehabilitation; robotic-assisted; synergy; motor control; motor learning; upper limb

\section{Background}

The expression Moving the Goal Posts (MGP) commonly refers to an unfair change to goals. However, such an approach can be used to progressively improve one's capabilities, when the limits of those capabilities are unknown. Examples of such approach can be seen in neuro-rehabilitation, where recovery is not achieved suddenly 
but rather progressively $[1,2]$. Exercises and tasks are thus defined progressively to encourage motor control changes. In this process, clinicians usually set a reachable goal and move it further and further to favour changes. This applies either to practiced tasks of increasing difficulties (e.g. Range of Motion or finer motor control) but also to the expected changes in motor behaviours (movement quality, limitation of over-recruitment, movement smoothness or movement speed). The MGP approach takes its theoretical basis in physiology. Brain physiology studies and plasticity theory show that the changes in neural configuration can happen only when the inputs to the neurons circuitry - and so the learning steps - falls within their anatomical available resources. This thus limits the learn-able step-size but does not ultimately prevent large scale changes if they are presented progressively [3].

\section{Previous works}

Robot-assisted training has shown great potential in neuro-rehabilitation [4] by enabling intensive motor therapy, which is a key factor of recovery [5]. Many different robotic strategies have been developed to that extent [6] but very little address the problem of the quality of movement [7]. As the stereotypical movement patterns, or pathological synergies, have a high prevalence in individuals post-stroke [8,9], movement pattern modification, or shaping, which aims to increase the quality of movement is an important and highly demanding application in neuro-rehabilitation. The shaping of these stereotypical movement patterns could prevent further complications and lead to better functional outcomes [10].

Among the few robotic-assisted training algorithms addressing this issue, the Time-Independent Functional Training (TIFT), proposed in 2011, provides realtime haptic feedback to the user by blocking hand movement when they are not moving with a specific joint coordination [11]. After the intervention, healthy subjects achieved significantly better results than other two groups. However, the training objective in this experiment was explicitly stated to the subjects. This limits the interpretation of the results, as they can be voluntarily influenced by the subjects and thus limits the possible translation to neuro-rehabilitation where individuals may not be able to freely adapt their movement pattern.

To that extent, implicit learning has been considered to be a more appropriate training method, potentially leading to a longer retention $[12,13]$. Implicit training of movement patterns was studied in Proietti et al. [14]. In this study, a Kinematic Synergy Controller (KSC) which applies torque constraints distributed on all the joints using an upper-limb exoskeleton when the movement pattern to be trained is undesired was used [15]. Healthy individuals were asked to complete a reaching task without information on the training goal and while the exoskeleton was applying the kinematic constraint [14]. The results showed that the approach can significantly change subjects' movement patterns which persist to an extent during the wash-out phase. However, KSC's main limitation is the requirement of a direct kinesthetic feedback at each considered joint. This approach could thus only be implemented on exoskeletons which usually have matched motor joints and physiological joints, at the cost of a higher complexity, cost and setup time.

Indirect Shaping Control (ISC) was thus proposed by the authors to address this limitation and extend the movement pattern modification algorithms to endeffector based devices - where users interact with the robotic device through one 
attachment point by holding onto the end-effector of the robot with their hand [16]. As opposed to KSC, the ISC has an indirect physical interaction with the movement pattern to be modified: a force field applied only in task space (i.e. at the hand) affects the redundant joint space (i.e. the swivel angle). The approach is based on the motor control theory which states that humans, when presented with a high level of redundancy to achieve a given task, attempt to minimise a cost, and thus resolve this redundancy $[17,18]$. Several models incorporating various costs have been proposed and, if there are still debates about the exactitude of each cost function independently, it is acknowledged that the optimisation account for a form of energy expenditure $[17,19,20]$.

In ISC, an artificial energy cost, in the form of a viscous force field that scales with the undesired movement pattern is added by the robotic device upon the effort of the human user at the attachment point. The cost to perform a movement with an undesired pattern is increased (i.e. a higher viscosity) through robotic intervention, while the cost decreases when the movement is closer to the desired movement pattern. Based on motor control theory [17], the trainee is expected to modify their movement pattern after unconscious exploration to reach a more optimal movement pattern with smaller overall cost. The limited results on five subjects of Fong et al. study [16] showed that healthy subjects could modify their movement pattern unconsciously through this indirect shaping strategy.

\section{Objective}

Despite these different movement shaping approaches, explicit or implicit, direct or indirect, the idea of inducing a change through a progressive goal is still missing in the literature. As discussed previously, whilst the process of neuronal dominance change limits the magnitudes of the learning step, a large-scale change may be achieved through a series of smaller learning steps [3]. In all previous movement shaping studies, a constant goal was set during the intervention phase. We hypothesize here that a moving goal would lead to a larger movement pattern modification than a constant goal. In this paper, the Indirect Shaping Control (ISC) was selected and employed as the movement shaping method, delivered through an end-effector based robot.

This hypothesis was tested in a randomised control single blind study, with 20 healthy participants, comparing the effect of the ISC with a constant goal, previously introduced, and the proposed modified ISC algorithm with a progressively increasing goal.

\section{Methodology}

Healthy subjects were asked to perform reaching tasks while attached to the EMU manipulandum applying the ISC. In the Control group, the goal of the ISC was set constant across the intervention (C-ISC) whereas in the MGP group the goal was increased progressively during the intervention (P-ISC). Comparisons on effectiveness in movement shaping and movement changes were made between the two groups. 


\section{Participants}

Twenty healthy subjects (age: $23.9 \pm 3.0$ ) participated in this experiment and were randomly allocated into two groups: the MGP group where the intervention used a Progressive-ISC (P-ISC) and the Control group with a Constant-ISC (C-ISC). Participants in the two groups shared the same setup and protocol except for this condition.

This experiment was approved by the University of Melbourne Human Research Ethics Committee (\#1749444) and an informed written consent was received from all subjects.

\section{Task design}

In this experiment, the objective of the robotic intervention was to increase the swivel angle with which the subjects were performing reaching tasks using their dominant hand. The swivel angle corresponds to the degree of redundancy of the human arm and is thus not directly controlled by an end-effector device, such as the EMU (see Figure 1) which only applies forces in 3 dimensions at the hand level. This task has been selected for its relevance to neuro-rehabilitation, as stroke survivors frequently demonstrate abnormal coupling of elbow and shoulder muscles. In this reaching task, their three heads of deltoid could be activated in one single synergy in an unnatural way which results in a stereotypical movement pattern with an exaggerated swivel angle $[9,21]$.

Participants were required to sit on a fixed chair and repeat a reaching movement from their lap to a touch screen positioned in front of them as shown on Figure 1. The distance and height of the touch screen was normalised for each subject such that the height of the upper edge of the touch screen aligned with their chin and that their metacarpophalangeal joint was touching the screen when they fully extend their arm.

During the experiment, in order to demonstrate implicit learning method, a quiz game was designed to distract the subjects from the exact objective of the study. The quiz User Interface (UI) was displayed on the touchscreen with the reaching target corresponding to the quiz answers buttons.

\section{Apparatus and measurement}

In this experiment, the EMU, which is a three-dimensional end-effector based rehabilitation robotic device [22], was used to generate a viscous force field to shape subjects' movements. The subject's hand held the handle of the device with their wrist strapped, preventing wrist flexion/extension and abduction/adduction (see Figure 1). This configuration allowed the device to produce a force interaction in $3 \mathrm{D}$ whereas the orientation of the hand was left free to rotate.

A trakSTAR 3D Guidance Magnetic Sensors (Ascension Technology Corporation, USA) was used to measure the subjects' shoulder $(S)$, wrist $(W)$ and elbow $(E)$ positions, which was subsequently used to calculate online the swivel angle as follows.

Vectors $\overrightarrow{S W}$ and $\overrightarrow{W E}$ were calculated using $S, E$ and $W$ positions and the normal vector to the SEW plane was expressed as:

$$
\mathbf{n}_{\text {arm }}=\frac{\overrightarrow{S W} \times \overrightarrow{W E}}{|\overrightarrow{S W}| \cdot|\overrightarrow{W E}|}
$$


where $\mid$ o| denotes the 2-norm.

Then, assuming the subjects maintained their trunk upright, the swivel angle can be calculated as:

$$
\theta=\arcsin \left(\mathbf{n}_{a r m} \cdot \mathbf{v}\right)
$$

with $\mathbf{v}$ an absolute vertical vector.

The EMU's real-time controller (an sbRIO-9637, National Instruments) was connected to a laptop which performed the force-field computation based on the swivel angle value and displayed the interface on a touch screen. All software was custom and written in LabVIEW.

Additionally, the swivel angle as well as the sensors positions and velocities (obtained through differentiation) were recorded during the movements.

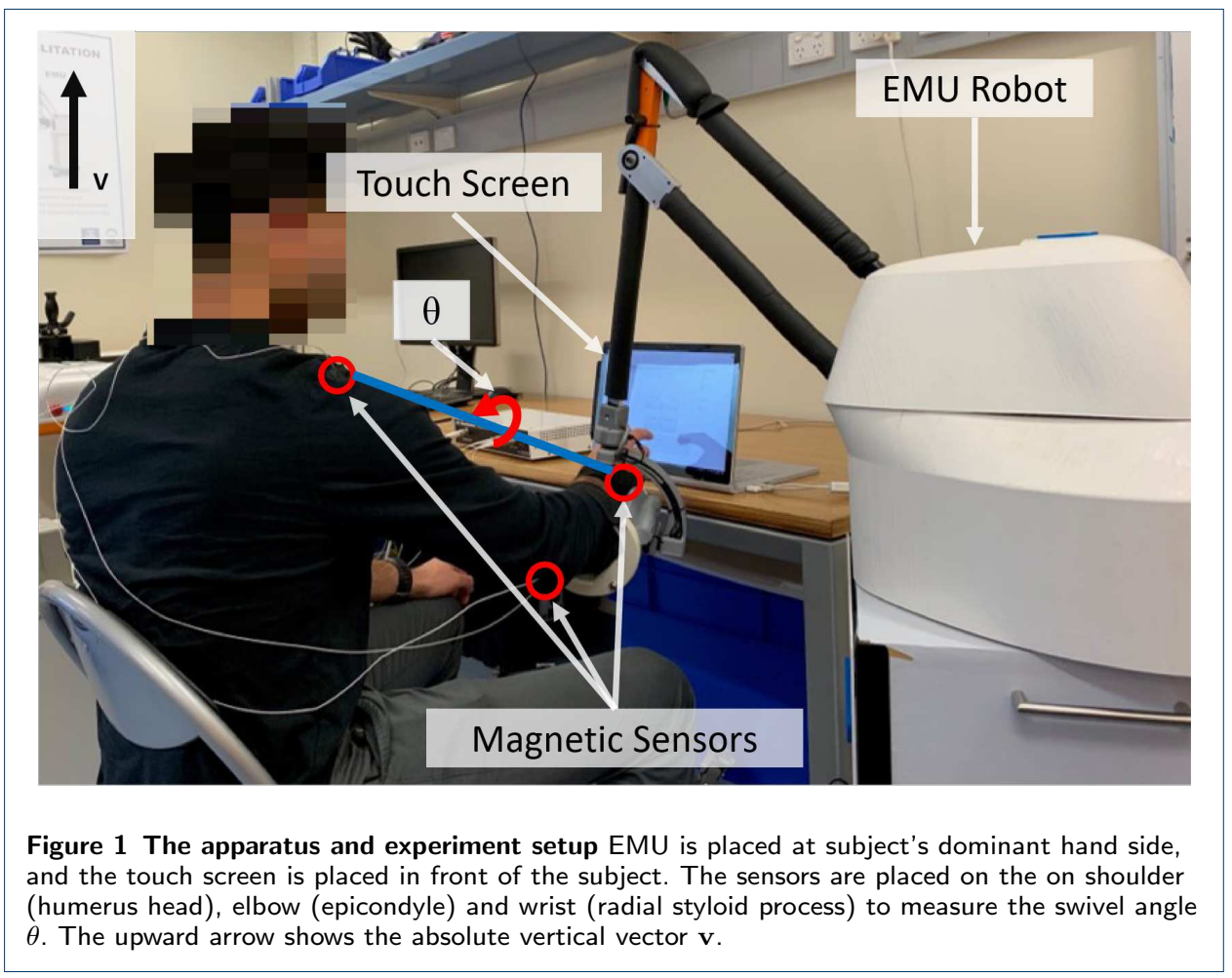

\section{Experiment protocols}

The experiment protocol was divided into five successive phases detailed below and summarized in Table 1.

Table 1 Experimental Protocol

\begin{tabular}{|c|c|c|c|c|c|}
\hline Phase & PRE-F & PRE-R & INT & POST-R & POST-F \\
\hline \multirow{2}{*}{ Robot Mode } & \multirow{2}{*}{ N.A. } & \multirow{2}{*}{ Transparent } & C-ISC & \multirow{2}{*}{ Transparent } & \multirow{2}{*}{ N.A. } \\
\cline { 4 - 4 } & & & P-ISC & & \\
\hline Iterations & $1-25$ & $26-50$ & $51-150$ & $151-175$ & $176-200$ \\
\hline
\end{tabular}

1 PRE-F: PRE-Free phase, where subjects performed 25 reaching tasks without being attached to the robot. Movements were recorded using the trakSTAR sensors, but data collected in this phase is not used in the current analysis. 
2 PRE-R: PRE-Robot phase, where subjects are strapped to the robot and performed another 25 reaching tasks. In this phase, the robot is set in transparent mode, compensating only for its own weight and friction, in order to minimise its influence on the subject. The movements recorded in this phase are used to obtain a reference for each subject.

3 INT: The INTervention phase, where subjects remained strapped to the robot and performed 100 reaching tasks. The robot applied either the "C-ISC mode" for the Control group or the "P-ISC mode" for the MGP group.

4 POST-R: POST-Robot phase, identical to the PRE-R phase, where subjects performed 25 reaching tasks with the robot set in transparent mode. This phase allowed to measure the washout effect.

5 POST-F: POST-Free phase, identical to PRE-F phase, where subjects are detached from the robot and performed another 25 reaching tasks with only the sensors recording. Data collected in this phase is not used in the current analysis.

Subjects performed a total of 200 movements. In order to reduce the potential influence of muscle fatigue, subjects were asked to take at least a thirty-second break every 20 iterations and could request additional rest at any time.

Subjects were not instructed at all of the different phases of the experiment and were blind to the objective of the experiment and to the effect of the robotic force field. Making the subjects unaware of the objective is required here to ensure that no active or conscious behaviour is influencing the results.

At the end of the experiments, participants were asked to take a questionnaire to test their awareness of the effect of the robotic device.

\section{Indirect Shaping Control (ISC)}

Indirect Shaping Control was previously introduced in [16]. Its principle is to apply a viscous field to the subject's hand movement, as a function of the current swivel angle value. This artificially increases the movement cost (i.e. increasing the viscosity) the further the movement pattern (here the swivel angle) is from a desired movement pattern. The force applied by the device at the subjects' hand is thus calculated as:

$$
\mathbf{f}_{\text {vis }}=\left\{\begin{array}{cl}
-b_{i} \cdot b_{k} \cdot \Delta \theta \cdot \dot{\mathbf{x}} & , \text { if } \Delta \theta>0 \\
0 & , \text { otherwise }
\end{array}\right.
$$

where

- $\mathbf{f}_{\mathbf{v i s}}$ is the force applied at the end effector;

- $\dot{\mathbf{x}}$ is the real-time hand velocity in $m \cdot s^{-1}$;

- $\Delta \theta=\theta_{\text {desired }}-\theta$ is the real-time difference between a desired swivel angle $\theta_{\text {desired }}$ and the measured swivel angle $\theta$ (in degrees);

- $b_{i}$ is a scalar factor changed according to the current iteration $i$ and which aims to introduce and remove the viscous field gradually during the INT phase. In this experiment, $b_{i}$ increases linearly from 0 to 1 in the first 15 iterations in INT phase and decreases linearly from 1 to 0 in the last 15 iterations in INT phase. $b_{i}$ remains at 1 in the other iterations; 
- $b_{k}$ is a tuning gain to make the force field within a reasonable range and has been empirically set to $5 \mathrm{~N} \cdot s \cdot\left(\mathrm{m}^{\circ}\right)^{-1}$ in the whole experiment for all subjects.

The force field thus relies on a swivel angle difference between $\theta_{\text {desired }}$ and measurement $\theta$. In order to take into account subject natural movement variability, the robotic generated desired movement pattern $\theta_{\text {desired }}$ was based on a subject original swivel angle over the course of movement, identified during the PRE-R phase. For each subject, a unique $\theta_{\text {original }}(d)$ was thus identified as a polynomial of degree three of the distance to the reaching point $(d)$. Additionally, in order to not influence the static posture of the subject at the initiation of movement $\left(d=d_{0}\right)$, the swivel angle alteration was designed to progressively changed along the movement reaching path, starting with the subject's natural posture and ending with the maximum change for the given iteration. Namely, the personalised desired swivel angle $\theta_{\text {desired }}$ in iteration $i$ and at a distance $d$ from the starting point was defined as:

$$
\theta_{\text {desired }}(d, i)=\theta_{\text {original }}(d)+\frac{d}{d_{\text {max }}} \cdot \theta_{\text {goal }}(i)
$$

where

- $d_{\max }$ is the distance from starting point to the button on the screen;

- $\theta_{\text {goal }}(i)$ is the shaping goal at $d_{\max }$

As shown in Table 1, two variations of the ISC controller were applied in the INT phase depending on the group. For the Control group, similarly as what proposed in $[16], \theta_{\text {goal }}(i)$ is set to a constant value of $10^{\circ}$ across all INT iterations. For the MGP group, $\theta_{\text {goal }}(i)$ was linearly increased from 0 to $10^{\circ}$ in the first 50 iterations and kept constant at $10^{\circ}$ for the last 50 iterations, as presented on Figure 2 (a). Thus, the $\theta_{\text {goal }}$ was the same for both groups in the second half of INT phase. Examples of evolution of $\theta_{\text {goal }}(i)$ along the movement path are presented for both cases on Figure 2 (b).

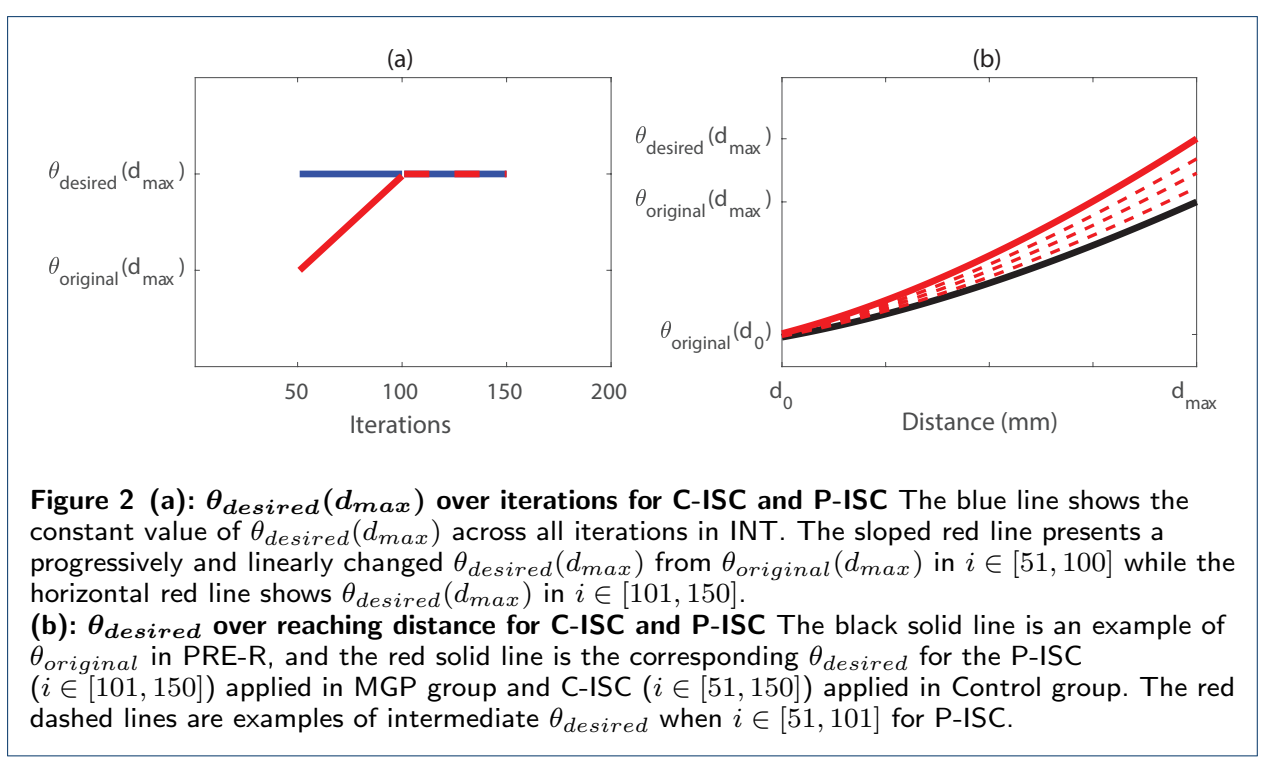




\section{Data analysis}

In this study, the viscous force and the swivel angle were calculated online using NI LabVIEW 2016 and the post-processing of the data and statistical analysis were performed using MATLAB 2019b.

\section{Questionnaire}

All subjects were asked to take a questionnaire which consisted of the following questions:

1 Question 1: Did you feel the robot applying any force?

2 Question 2: Do you think the robot was influencing your movement in a particular way? If yes, what was that influence?

3 Question 3: Do you think you changed the way you move during the experiment? If yes, how did you change the way you move?

\section{Shaping outcome}

To evaluate the effect of the intervention, the primary measure is the swivel angle in the different phases of the experiment. As the desired swivel angle was progressively increased along the trajectory, only the value at the end pose $\left(d=d_{\max }\right)$ was used in the analysis. For each group, this measure is thus reported, averaged for each subject:

- during the PRE-R phase (iterations $i=26$ to $i=50$ ), used as a baseline;

- during the INT phase, for the iterations $i=101$ to $i=135$ where both groups share the same shaping goal with $b_{i}=1$, to investigate the immediate effect of the ISC compared with baseline for the two groups;

- during the POST-R phase (iterations $i=151$ to $i=175$ ), to investigate the retention effect compared with baseline.

Comparison between the phases for all subjects was further tested using a Wilcoxon signed-rank test.

Additionally, to compare the two variations of the ISC, the average value $\overline{\theta^{P R E-R}}\left(d_{\max }\right)$ recorded during the PRE-R phase for each subject was subtracted to the measures obtained in each subsequent phase to obtain the true change of swivel angle induced by the intervention, independently of the between subjects variability.

Similarly, the effect of the intervention was also assessed for each subject individually.

\section{Error evolution}

To more specifically investigate the effect of the MGP approach and observe the adherence of the subjects to the current goal, the $\theta_{\text {error }}$ measure was introduced:

$$
\theta_{\text {error }}=\Delta \theta\left(d_{\max }\right)
$$

It represents the compliance of the subject to the current algorithm objective, which is progressive in the MGP group and constant in the Control group. Similar to previous measures, only the swivel angle at end pose of each iteration were compared, for every iteration in which $b_{i}=1(i=66$ to $i=135)$. 
Hand velocity

A last measure, $\phi$, was introduced to evaluate the potential effect of the force field on subjects' movements "strategy". Indeed, given that the proposed artificial cost introduced by the ISC is based on the movement velocity, a change in the velocity could explain a different optimisation from the subject to counteract this artificial cost. The average velocities of each iteration, $\overline{\left|\dot{\mathbf{x}}^{\{P H A S E\}}\right|}$, was obtained after position differentiation and the average over the different phases was then calculated. A coefficient of velocity change between PRE-R and INT phase was then obtained for each subject as:

$$
\phi=\frac{\left(\overline{\left|\dot{\mathbf{x}}^{I N T}\right|}-\overline{\left|\dot{\mathbf{x}}^{P R E-R}\right|}\right)}{\overline{\left|\dot{\mathbf{x}}^{P R E-R}\right|}} .
$$

\section{Retention}

Identical to shaping outcome, for each group, this measure is thus reported, averaged for each subject:

- during the PRE-R phase (iterations $i=26$ to $i=50$ ), used as a baseline;

- during the POST-R phase (iterations $i=151$ to $i=175$ ), to investigate the retention effect compared with baseline.

Comparison between the phases for all subjects was further tested using a Wilcoxon signed-rank test.

\section{Results}

Results are presented in three parts, respectively presenting the subject awareness of the robotic effect, the direct intervention effect on subjects' movements and the retention effect during the washout period.

\section{Questionnaire results}

According to answers to the questionnaire, all of the subjects felt a force field was applied by the device (Q1). Only one subject suspected that the device influenced them in a particular way but was incapable of describing the actual effect (Q2). This subject also pointed out that he felt he changed his movement to "a parabolic trajectory" during the experiment, whereas all the other subjects did not feel they change their movement patterns (Q3).

\section{Shaping outcome}

The outcomes for the two groups are shown in Figure 3, with $\theta\left(d_{\max }\right)$, averaged for each subject, showed for PRE-R and INT phases in each group. In the Control group the median difference observed between PRE-R and INT phases was of $4.0^{\circ}$ $(p<0.05)$. In the MGP group the effect was larger with $6.2^{\circ}(p<0.05)$.

Figure 4 shows the change of swivel angle in the two groups during the second part of INT phase $(i=66$ to $i=135)$ relative to baseline during PRE-R. The difference between the two groups in the INT phase was small, at $1.4^{\circ}$ but observed to be significant $(p<0.001)$.

The change of swivel angle for each individual subject is shown in Figure 5. All subjects but one $(\mathrm{S} 1)$ had a significant increase of swivel angle $(p<0.05)$. S1 showed 


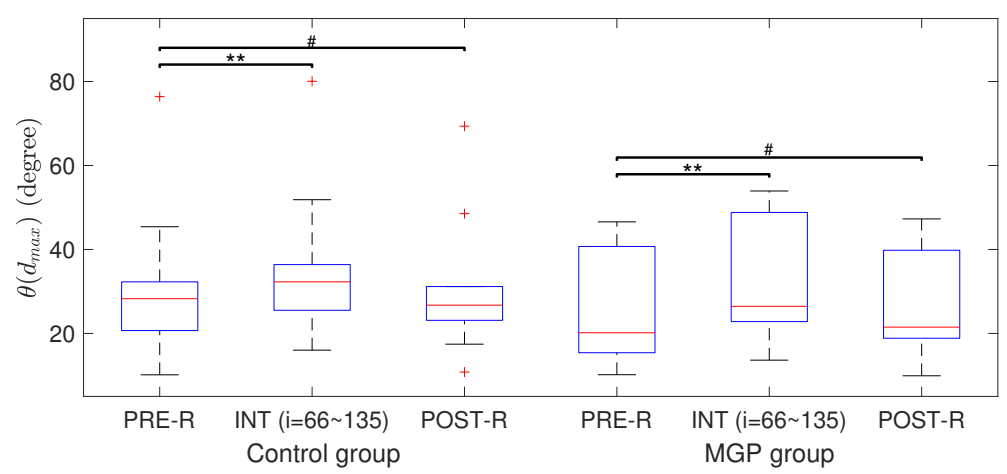

Figure 3 Intervention and Retention outcomes for each group Bottom and top edges of the blue box indicate the 25 th and 75 th percentiles respectively while the red + shows the outliers. Inside the blue box, the red solid line shows the median of the data set. \#: $p \geq 0.05, *: p<0.05, * *$ : $p<0.01, * * *: p<0.001$.

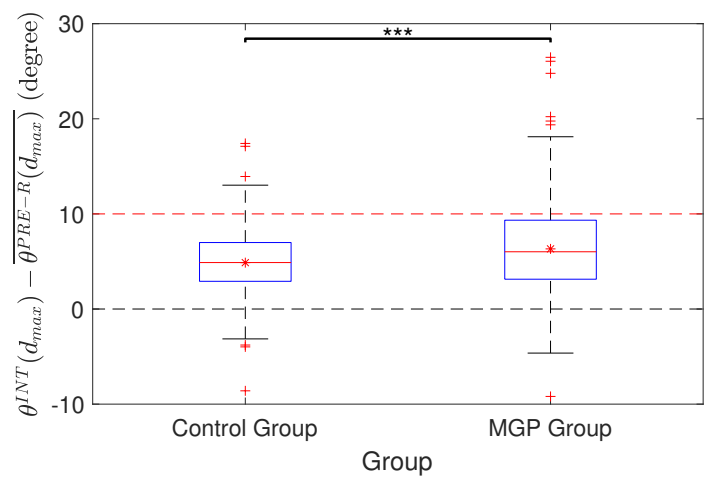

Figure 4 Intervention outcomes for the two groups The box plot shows intervention outcomes (iterations 101 to 135) for each group. Bottom and top edges of the blue box indicate the 25th and 75 th percentiles respectively while the red + shows the outliers. Inside the blue box, the red solid line shows the median and the red star shows the mean value of the data set. *: $p<0.05$, $* *: p<0.01,{ }^{* * *}: p<0.001$.

only a very small, non significant change of less than $1^{\circ}$. All others demonstrated a change included between $3^{\circ}$ and the goal value of $10^{\circ}\left(\theta_{\text {goal }}\right)$, except for one subject (S16) slightly overshooting the goal.

\section{Error evolution}

The evolution of $\theta_{\text {error }}$ for each group is shown in Figure 6 . The average of mean values across all iterations is $-3.2^{\circ}$ for MGP group and $-5.3^{\circ}$ for the Control group. No clear trend of evolution can be observed. However, the error was observed to be consistently lower in the MGP group than in the Control group across the entire iteration and not only during the last part of INT phase $(i=66$ to $i=135)$ as reported in the previous section.

\section{Hand velocity}

The velocity change ratio $\phi$ for each group is shown in Figure 7. A reduction of the average hand velocity was observed in both groups, with a coefficient of 0.19 and 


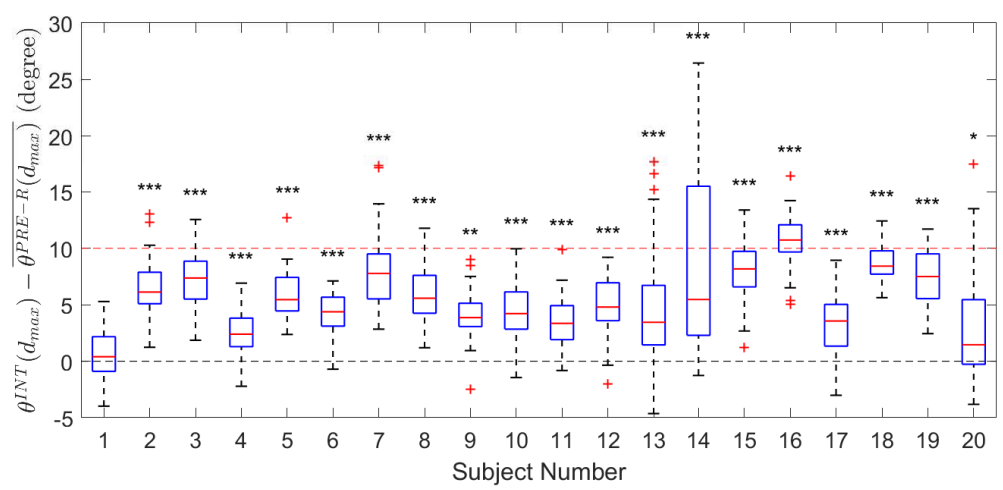

Figure 5 Individual intervention outcomes The box plot shows individual intervention outcomes (iterations 101 to 135). Subjects 1 to 10 were in Control group and Subjects 11 to 20 were in MGP group. Bottom and top edges of the blue boxes indicate the 25th and 75th percentiles respectively while the red + shows the outliers outside the box and the red solid line shows the median value inside the blue box. Significance is reported compared to PRE-R. *: $p<0.05,{ }^{* *}$ : $p<0.01$, ***: $p<0.001$.

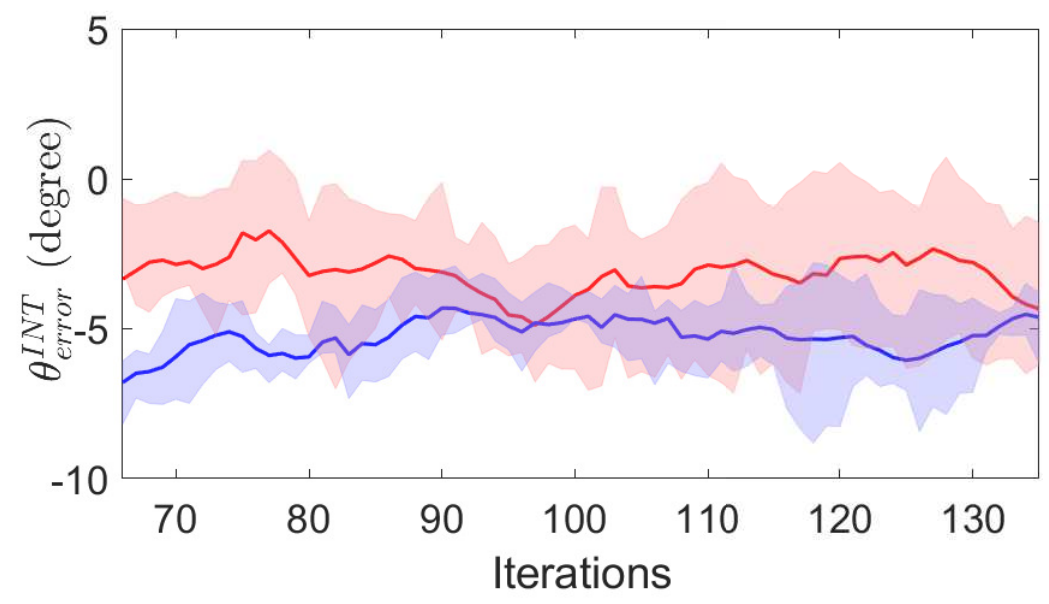

Figure 6 Error over INT phase for the two groups The red and blue solid line shows the mean error in each iteration for all subjects in MGP group and Control group respectively. Shaded areas represent the interquartile range after rejecting outliers (three-sigma rule).

0.14 for the Control group and the MGP group respectively. The difference between the two groups, 0.05 , was shown to be significant $(p<0.001)$.

\section{Retention}

The retention effect is evaluated by comparing the $\theta\left(d_{\max }\right)$ between PRE-R and POST-R phases and showed on Figure 3. No significant difference is found for either group between PRE-R and POST-R. 


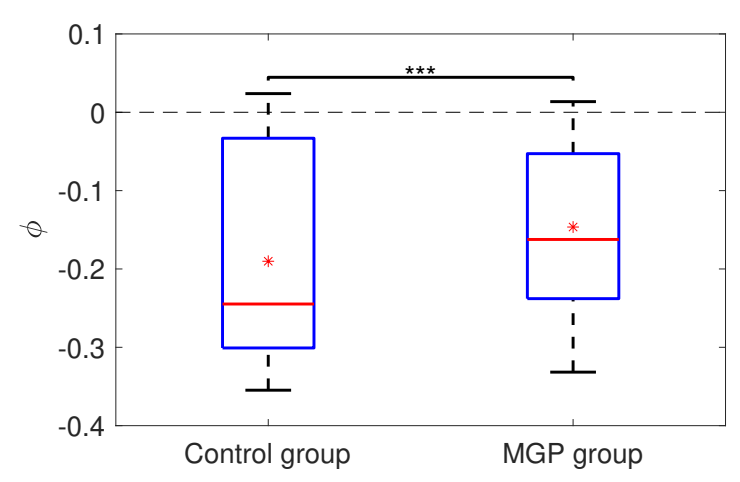

Figure 7 Velocity changes ratio from two groups The box plot shows velocity changes ratio $\phi$ in INT phase for Control group and MGP group. The bottom and top edges of the blue box indicate the 25 th and 75 th percentiles respectively while the red + shows the outliers. Inside the blue box, the red solid line shows the median and the red star shows the mean value of the data set. *: $p<0.05, * *: p<0.01, * * *: p<0.001$.

\section{Discussion}

Movement pattern modification

Indirect Shaping Control outcomes

A significant change in movement pattern was observed in both groups per their swivel angle in the second half of the INT phase $(i \in[101,135])$. Although the changes remain of relatively small amplitude $\left(4^{\circ}\right.$ and $6^{\circ}$ respectively) this demonstrates the possibility that motor behaviour can be influenced in a desired "direction" (i.e. an increase of the reaching swivel angle) without direct physical intervention and without explicit instructions given to the subjects. It is important to note that every subject demonstrated a change in the expected "direction". These results strengthen the ISC approach introduced by the authors in [16] and where limited results were presented (two out of five subjects changed their movement pattern into another direction). The initial experiment and the Control group shared the same apparatus and setup but with two differences which may explain the different results. The first change is that in the previous work, the velocity measurement and physical contact point were at the center of the wrist. The present work changed this to the center of the hand. Thus, the previous version potentially prevented a fully free movement around the swivel angle for the subjects, due to this physical constraint at the wrist. The second change is the measurement of $\theta_{\text {original }}$ which is here modeled as a polynomial instead of taken as a linear relationship on constant mean value in the previous work. Thus, the previous work may have led to a more artificial linear swivel angle change along the trajectory, while the current implementation better respects the natural movement pattern reference along the path, only influencing a shift of this value.

\section{Contribution of Moving the Goal Post approach}

The MGP approach evaluated here is shown to be more effective in this context than its equivalent with a constant goal across the training. Although the difference between the two groups appears relatively small $\left(1.4^{\circ}\right)$, it is significantly detectable and corresponds to an increase of $29 \%$ over the Control group. This confirm the 
initial hypothesis that a moving goal would lead to a larger movement pattern modification compared to a constant goal one when used in an indirect and implicit shaping intervention. In more detail, similar effects can be observed from the error evolution over the iterations (see Figure 6). Although no clear trend of error evolution in each iteration could be observed, it can be seen that the moving goal leads to a smaller mean error in most iterations, suggesting a better compliance of the subjects with the MGP approach.

Motor cost influence on movement strategy

In ISC, the artificial additional cost introduced is in the form of a viscous force opposed to the movements in the direction of the hands velocity, a higher velocity causing higher viscous force magnitude. The viscous force field is proportional to both swivel angle error and velocity (as per Equation 3) and the subjects could thus choose to either comply to the swivel angle requirement or reduce their reaching velocity to reduce the intensity of the force field. In Figure 7, it can be clearly seen that subjects in both groups slightly reduce their velocity magnitude to avoid the resistance in completing the task in the majority of iterations.

Due to the nature of the task, the natural motor cost is here dominated by the gravitational load, which is static by essence: a higher swivel angle will lead to a larger load on shoulder muscles for a given posture. A slower movement will require additional energy, as the load will have to be sustaining for a longer time. But limiting the speed of movement could reduce the artificial cost which is velocity dependent. The cost introduced by the ISC thus counteracts this effect and a compromise between these two costs is expected to be found by the subjects. This can explain the reduction in the hand velocity and the compliance of the subjects to the modification of their swivel angle, suggesting an exploration of the cost space by the subjects.

It is also to note that, as for the compliance to the required swivel angle goal, there is a significant difference between the two groups in their change of velocity. Comparing with the velocity in PRE-Robot phase, the average velocity dropped by $19 \%$ for the Control group and $14 \%$ for the MGP group in INT phase, comforting this analysis. The Control group subjects tend to explore and comply less with the goal but to favour a velocity reduction.

\section{Retention}

No retention can be observed in either group, suggesting that the subjects quickly came back to their original movement pattern when the force field was removed. Despite the application of an implicit approach, supposedly leading to better retention, in this study, long term retention was not expected to happen, as the desired exaggerated new movement pattern leads to a higher cost when the artificial cost is removed, due to the existence of gravity. Subjects thus do not gain any benefit from this new movement pattern when they are moving without the artificial force field.

This minimal retention effect differs from the results observed by Proietti et al. where a direct, but implicit, movement shaping is provided with an exoskeleton [14]. In their work, a direct torque was applied on the corresponding joints to shape the 
joints' coordination. The retention observed in their experiment could be due to the larger effect observed on their subjects at the end of the intervention phase, leading to a longer washout.

Additionally, in [14], the subjects' awareness was not checked after the experiment making it difficult to fully conclude on whether the subjects voluntarily adapted to the force field, or were conditioned to move in a certain way when placed in the test setup.

\section{Subject awareness}

From the questionnaire results, the subject awareness can be evaluated. All participants were asked to focus on finishing the quiz and reaching tasks. As all participants could not describe the actual effect after experiment, the training can be seen as truly implicit.

A special case among them is Subject 14 who pointed out that a changed of his movement pattern occurred during the experiment and whose shaping outcome is one of the largest observed across the subjects (see Figure 5). It can be seen that the values of his swivel angle during the second part of the INT phase $(i \in[101,135])$ have a significant wider variation compared with the other subjects. This variation shows a larger exploration of the cost space and potentially demonstrates that the subject noticed the force field and explicitly changed their movement pattern during the experiment. Interestingly, even though the subject was not able to describe the actual intervention effect, they still found the way to reduce the artificial cost by complying to the desired movement pattern.

\section{Translation to neuro-rehabilitation}

The chosen task and problem in this study aims to be relevant to motor neurorehabilitation of the Upper-Limb (UL), where pathological synergies retraining and movement correction play an important role towards functional recovery. This study aimed to provide a method in adapting human's movement pattern by progressively adding an indirect force field. The results demonstrate the feasibility of changing the joint space coordination by using a manipulandum device by adding an artificial task space cost. Specifically, the results demonstrate that when doing movement pattern modification with a progressive approach, as classically done in neuro-rehabilitation, updating the goal - or objective - of the intervention by small changes leads to larger scale changes. The fact that this approach is suitable for indirect methods such as the one presented in this work is particularly relevant to translation to neuro-rehabilitation. Indeed, compared to the previous work using exoskeletons [11, 14], this approach allows the use of much simpler and accessible devices for the same objective. But despite cost and practicality, the indirect approach could also have the additional benefit of actually forcing the subjects to completely adopt the movement pattern as no direct physical force constrains it. The effect that is observed in the redundant space to be shaped is truly due to the subjects' neurophysiological system, and not a combination of the robotic and the subject inputs as in the case of the KSC implementation [15]. This thus may lead to a more direct translation outside of the training setup as the motor pattern generated by the subject within the setup and outside the setup will result in the same movement outcome in both cases. 
On the limited retention observed in the current experiment, it might not be an actual limitation observed in a neuro-rehabilitation. Indeed, in the current scenario, the newly learned movement pattern does not bring any benefit to the subjects, whereas it is expected to be beneficial in the case of neurologically impaired subjects, and thus could encourage a longer and higher retention.

Finally, on the implicit aspect of the study, it is important to note that here the healthy subjects are physically easily capable of complying with the objective. If the subjects were explicitly described the desired movement pattern as well as the study objective, the results would be affected by how much the subjects want to cooperate the researchers. In fact, the implicit learning approach, even if suspected to lead to better retention, may not be possible or practical in a neuro-rehabilitation context where subjects may have little movement variability of movement to even explore the cost, and thus comply to it [23]. In any case, if the shaping component of the training is left implicit, it is important, as suggested in [14] to keep another reward mechanism. This mechanism can be task, and not shaping, related and classically in the form of gaming and/or a score to ensure motivation as well as favour dopamine release to promote brain plasticity [3].

\section{Limitations}

It could be seen that the average shaping outcome from both groups cannot achieve the shaping goal, at which the artificial component in the cost function could become zero. It is obvious that in this experiment, except the artificial force field, the movement is also dominated by the gravity which result in higher energy consumption and higher cost, when subjects were intended to achieve higher swivel angle. In this case, the actual overall optimum is slightly smaller than the desired movement pattern, which is similar to what we observed in the results of this experiment. Because the internal cost function of human is still in research, we cannot control exactly where we move the subject's movement pattern using ISC. Additionally, the results, with relatively small but significant effect, suggest that it is worth investigating longer training phases and training phases with steeper goal progressivity to determine whether it is possible to obtain larger changes.

The moving goal defined in this experiment was a linearly changed goal which aims to change the environment step by step. Although the overall performance of the MGP group is better than Control group, the shaping outcomes still varied person to person. In this case, adding personalised feedback could potentially contribute to the movement pattern modification. For example, slower or pausing the movement of the goal when trainees are not able to achieve it. In clinical application, this personalisation is common, as clinicians usually offer different treatments to different patients in different stages. However, if personalised feedback is integrated into ISC, it is important to ensure that this less challenging goal does not induce slacking which may reduce human effort during rehabilitation training and cause significant reduction in the outcome of the shaping [24].

\section{Conclusion}

In this randomised control single blind study, we investigated the contribution of a moving goal in promoting movement pattern modification by using two variations of 
an Indirect Shaping Control (ISC). Results show that all subjects, with or without a Moving Goal Post (MGP) approach, did adapt their movements towards the required movement pattern when trained using the robotic manipulandum, but no significant retention effect was observed in any of two groups. More importantly, a significantly larger adaptation was observed in the MGP group. These results extend the previous preliminary conclusions on indirect movement pattern modification using ISC [16] and show promise of both an indirect shaping approach for UL motor neuro-rehabilitation and the importance to an MGP approach in this context to maximise outcomes.

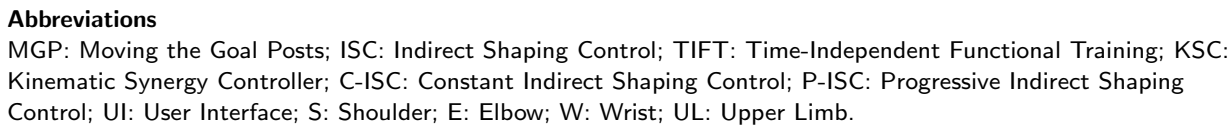

1. Lee TD, W.L.R.: Motor Learning Conundrums (and Possible Solutions). Quest 57(1), 67-78 (2005)

2. Winstein, C.J., Kay, D.B.: Translating the science into practice: Shaping rehabilitation practice to enhance recovery after brain damage. Progress in Brain Research 218, 331-360 (2015). Chap. 16. doi:10.1016/bs.pbr.2015.01.004

3. Nahum, M., Lee, H., Merzenich, M.M.: Principles of neuroplasticity-based rehabilitation. Progress in Brain Research 207, 141-171 (2013). Chap. 6. doi:10.1016/B978-0-444-63327-9.00009-6

4. Mehrholz, J., Pohl, M., T, P., Kugler, J., Elsner, B.: Electromechanical and robot-assisted arm training for improving activities of daily living, arm function, and arm muscle strength after stroke (Review). Cochrane Database of Systematic Reviews (9) (2018). doi:10.1002/14651858.CD009645.pub3.www.cochranelibrary.com

5. Sivenius, J., Pyörälä, K., Heinonen, O.P., Salonen, J.T., Riekkinen, P.: The significance of intensity of rehabilitation of stroke-a controlled trial. Stroke 16(6), 928-931 (1985). doi:10.1161/01.STR.16.6.928

6. Basteris, A., Nijenhuis, S.M., Stienen, A.H.A., Buurke, J.H., Prange, G.B., Amirabdollahian, F.: Training modalities in robot-mediated upper limb rehabilitation in stroke: A framework for classification based on a systematic review. Journal of NeuroEngineering and Rehabilitation 11(1), 1-15 (2014) doi:10.1186/1743-0003-11-111

7. Jarrassé, N., Proietti, T., Crocher, V., Robertson, O., Sahbani, A., Morel, G., Roby-Brami, A.: Robotic exoskeletons: A perspective for the rehabilitation of arm coordination in stroke patients. Frontiers in Human Neuroscience 8(947), 1-13 (2014). doi:10.3389/fnhum.2014.00947

8. Roby-Brami, A., Feydy, A., Combeaud, M., Biryukova, E.V., Bussel, B., Levin, M.F.: Motor compensation and recovery for reaching in stroke patients. Acta Neurologica Scandinavica 107(5), 369-381 (2003). doi:10.1034/j.1600-0404.2003.00021.x

9. Roh, J., Rymer, W.Z., Perreault, E.J., Yoo, S.B., Beer, R.F.: Alterations in upper limb muscle synergy structure in chronic stroke survivors. Journal of Neurophysiology 109(3), 768-781 (2013). doi:10.1152/jn.00670.2012

10. Levin, M.F.: Should stereotypic movement synergies in hemiparetic patients be considered adaptive? Behavioral and Brain Sciences 19(1), 79-80 (1996). doi:10.1017/s0140525×00041613 
11. Brokaw, E.B., Murray, T., Nef, T., Lum, P.S.: Retraining of interjoint arm coordination after stroke using robot-assisted time-independent functional training. Journal of Rehabilitation Research and Development 48(4), 299-316 (2011). doi:10.1682/JRRD.2010.04.0064

12. Gentile, A.M.: Movement science: Implicit and explicit processes during acquisition of functional skills. Scandinavian Journal of Occupational Therapy 5(1), 7-16 (1998). doi:10.3109/11038129809035723

13. Patton, J.L., Mussa-Ivaldi, F.A.: Robot-Assisted Adaptive Training: Custom Force Fields for Teaching Movement Patterns. In: IEEE Transactions on Biomedical Engineering, vol. 51, pp. 636-646 (2004). doi:10.1109/TBME.2003.821035

14. Proietti, T., Guigon, E., Roby-Brami, A., Jarrassé, N.: Modifying upper-limb inter-joint coordination in healthy subjects by training with a robotic exoskeleton. Journal of NeuroEngineering and Rehabilitation 14(1), 1-19 (2017). doi:10.1186/s12984-017-0254-x

15. Crocher, V., Sahbani, A., Robertson, J., Roby-Brami, A., Morel, G.: Constraining upper limb synergies of hemiparetic patients using a robotic exoskeleton in the perspective of neuro-rehabilitation. IEEE Transactions on Neural Systems and Rehabilitation Engineering 20(3), 247-257 (2012). doi:10.1109/TNSRE.2012.2190522

16. Fong, J., Crocher, V., Tan, Y., Oetomo, D.: Indirect Robotic Movement Shaping through Motor Cost Influence. In: IEEE International Conference on Rehabilitation Robotics, pp. 977-982 (2019). doi:10.1109/icorr.2019.8779430

17. Guigon, E., Baraduc, P., Desmurget, M.: Computational motor control: Redundancy and invariance. Journal of Neurophysiology 97(1), 331-347 (2007). doi:10.1152/jn.00290.2006

18. Todorov, E.: Optimality principles in sensorimotor control. Nature Neuroscience 7(9), 907-915 (2004). doi:10.1038/nn1309

19. Uno, Y., Kawato, M., Suzuki, R.: Formation and control of optimal trajectory in human multijoint arm movement. Biological Cybernetics 61(2), 89-101 (1989). doi:10.1007/BF00204593

20. Kang, T., He, J., Tillery, S.I.H.: Determining natural arm configuration along a reaching trajectory. Experimental brain research. Experimentelle Hirnforschung. Expérimentation cérébrale 167(3), 352-361 (2005). doi:10.1007/s00221-005-0039-5

21. Dewald, J.P.A., Beer, R.F.: Abnormal joint torque patterns in the paretic upper limb of subjects with hemiparesis. Muscle and Nerve 24(2), 273-283 (2001) doi:10.1002/1097-4598(200102)24:2¡273::AID-MUS130¿3.0.CO;2-Z

22. Fong, J., Crocher, V., Tan, Y., Oetomo, D., Mareels, I.: EMU: A transparent 3D robotic manipulandum for upper-limb rehabilitation. IEEE International Conference on Rehabilitation Robotics, 771-776 (2017). doi:10.1109/ICORR.2017.8009341

23. Levin, M.F.: Interjoint coordination during pointing movements is disrupted in spastic hemiparesis. Brain 119(1), 281-293 (1996). doi:10.1093/brain/119.1.281

24. Casadio, M., Sanguineti, V.: Learning, retention, and slacking: A model of the dynamics of recovery in robot therapy. IEEE Transactions on Neural Systems and Rehabilitation Engineering 20(3), 286-296 (2012). doi:10.1109/TNSRE.2012.2190827 


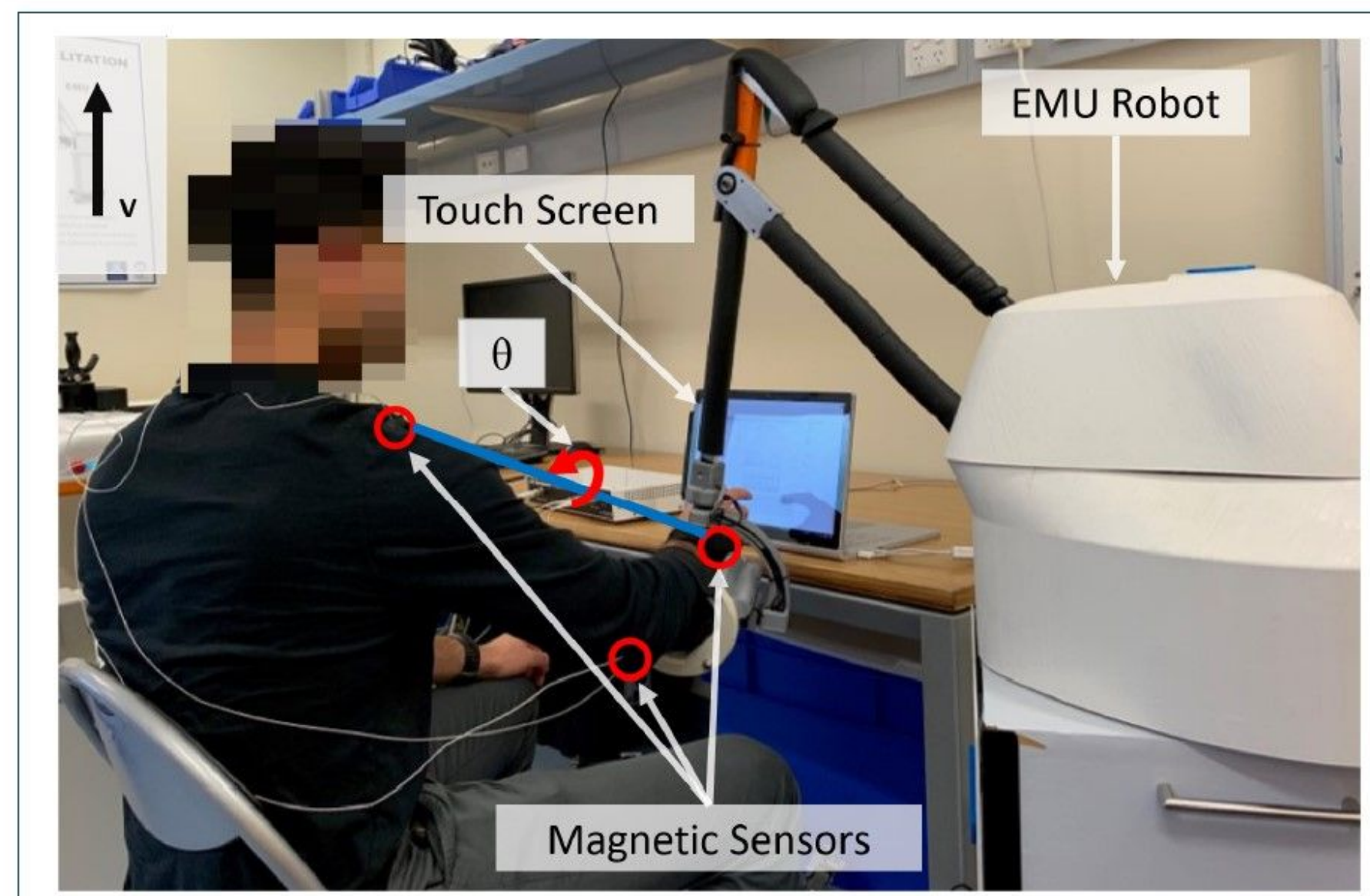

Figure 1 The apparatus and experiment setup EMU is placed at subject's dominant hand side, and the touch screen is placed in front of the subject. The sensors are placed on the on shoulder (humerus head), elbow (epicondyle) and wrist (radial styloid process) to measure the swivel angle $\theta$. The upward arrow shows the absolute vertical vector $\mathbf{v}$.

Figure 1 


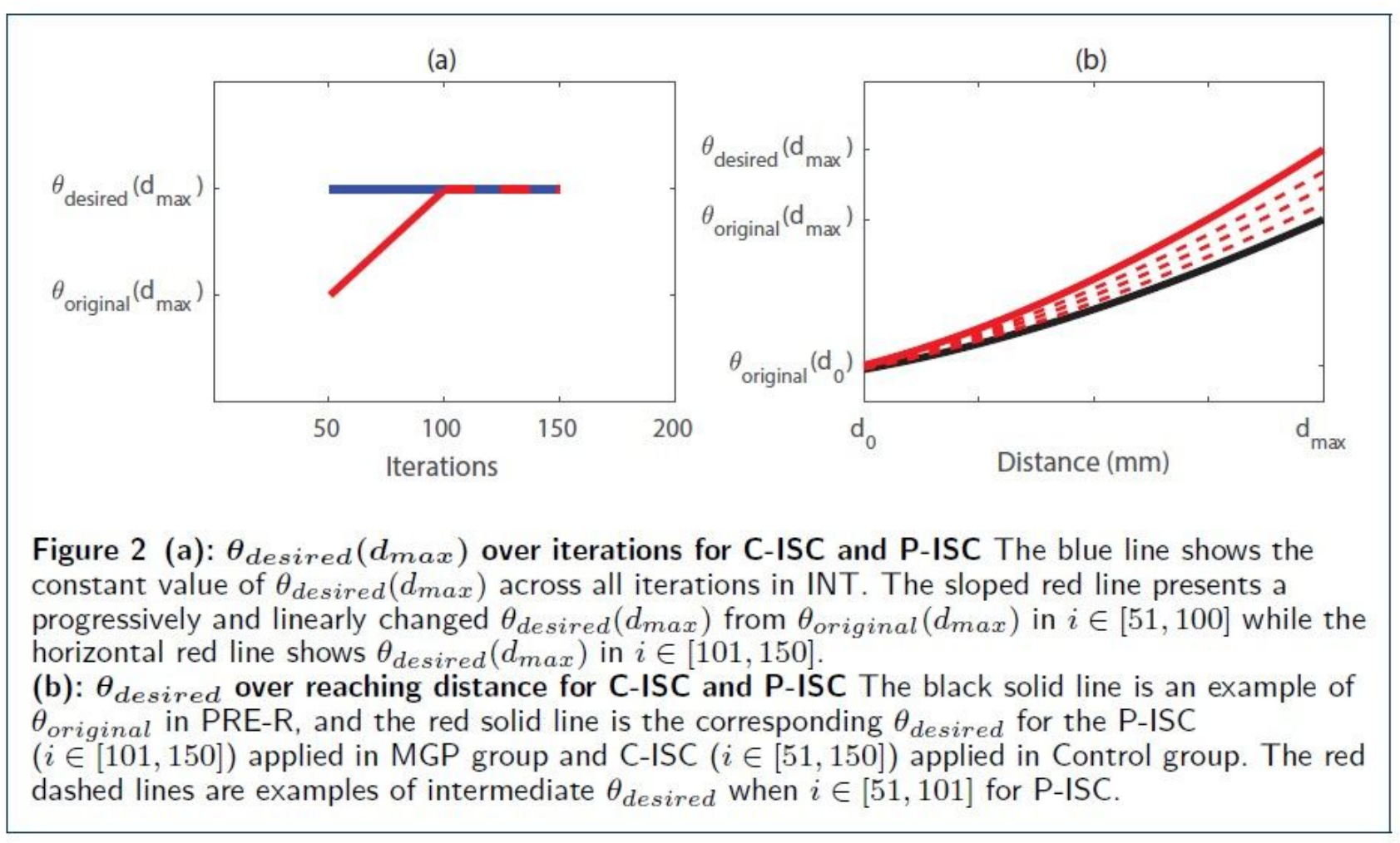

\section{Figure 2}

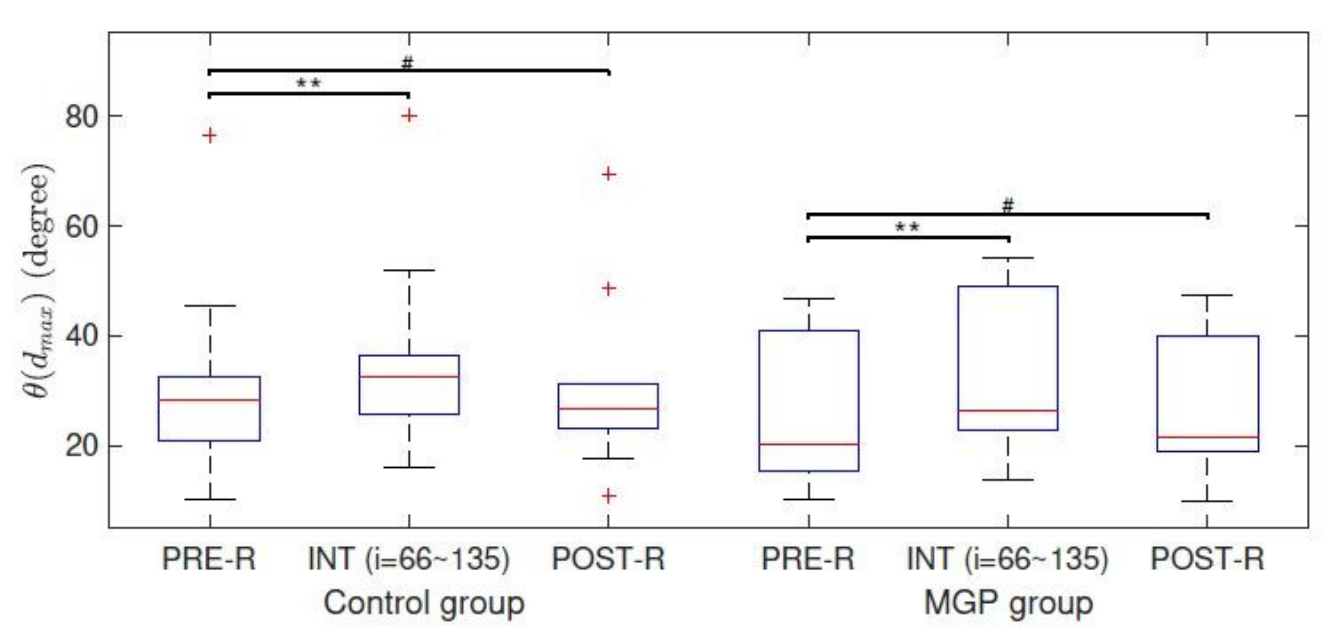

Figure 3 Intervention and Retention outcomes for each group Bottom and top edges of the blue box indicate the 25 th and 75 th percentiles respectively while the red + shows the outliers. Inside the blue box, the red solid line shows the median of the data set. \#: $p \geq 0.05, *: p<0.05, * *$ : $p<0.01,{ }^{* * *}: p<0.001$.

\section{Figure 3}




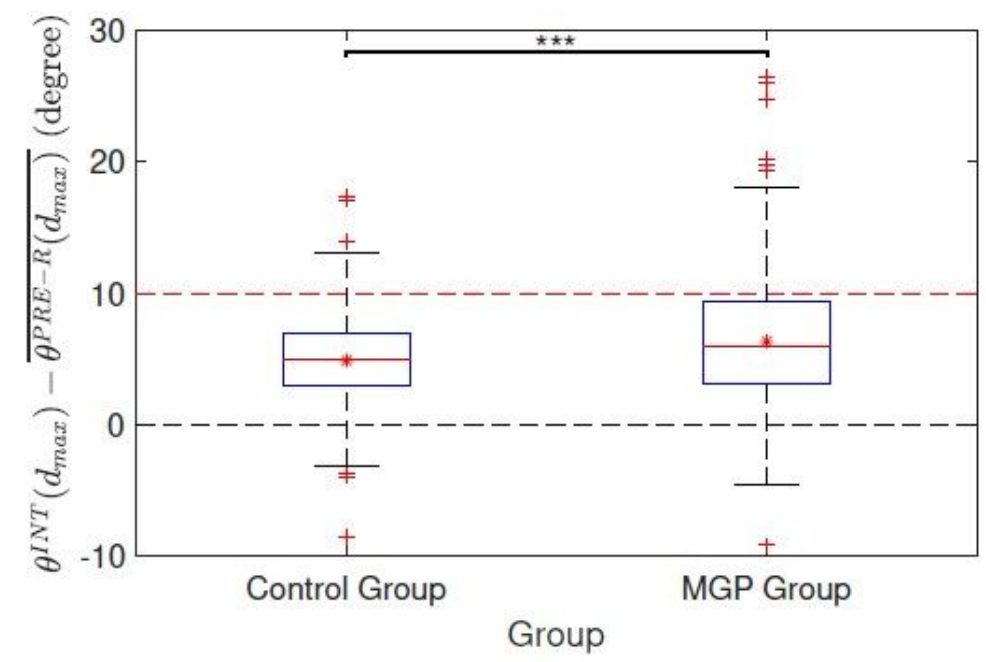

Figure 4 Intervention outcomes for the two groups The box plot shows intervention outcomes (iterations 101 to 135) for each group. Bottom and top edges of the blue box indicate the 25th and 75 th percentiles respectively while the red + shows the outliers. Inside the blue box, the red solid line shows the median and the red star shows the mean value of the data set. $*: p<0.05$, $* *: p<0.01, * * *: p<0.001$.

\section{Figure 4}

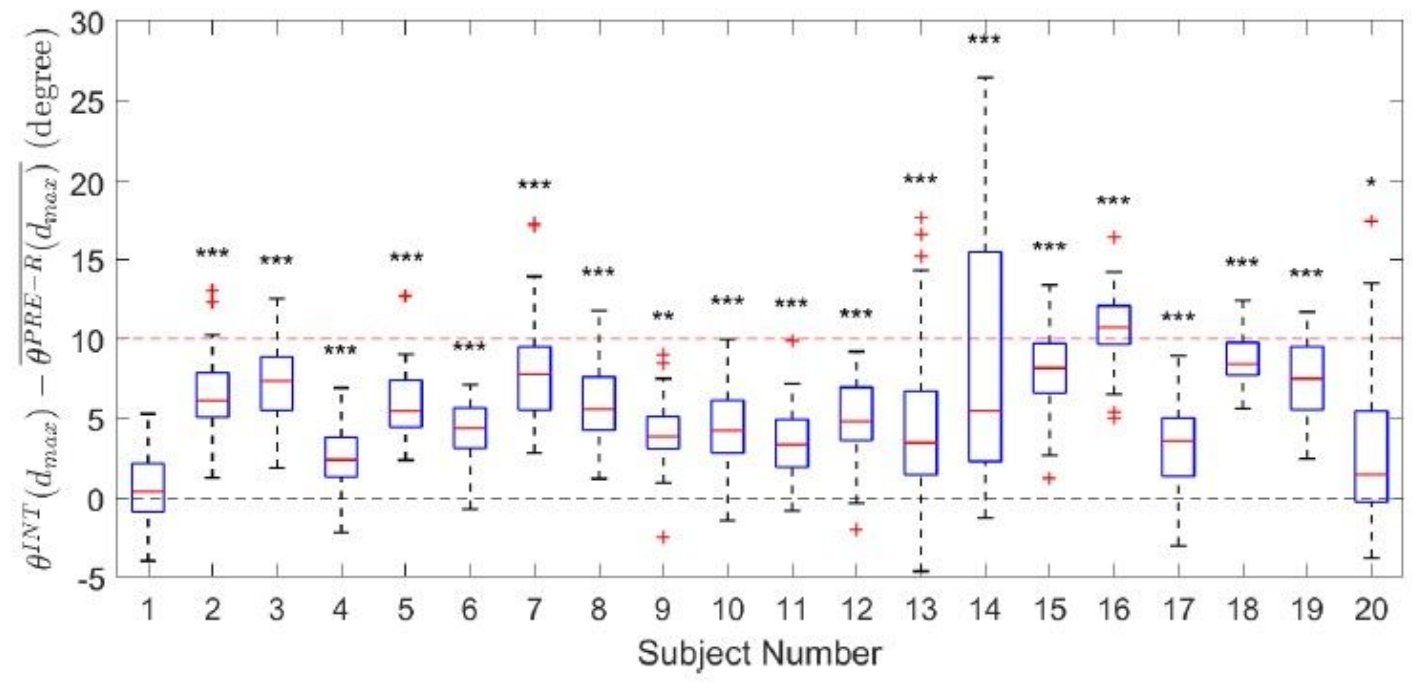

Figure 5 Individual intervention outcomes The box plot shows individual intervention outcomes (iterations 101 to 135). Subjects 1 to 10 were in Control group and Subjects 11 to 20 were in MGP group. Bottom and top edges of the blue boxes indicate the 25th and 75 th percentiles respectively while the red + shows the outliers outside the box and the red solid line shows the median value inside the blue box. Significance is reported compared to PRE-R. $*: p<0.05, * *$ : $p<0.01,{ }^{* * *}: p<0.001$. 
Figure 5

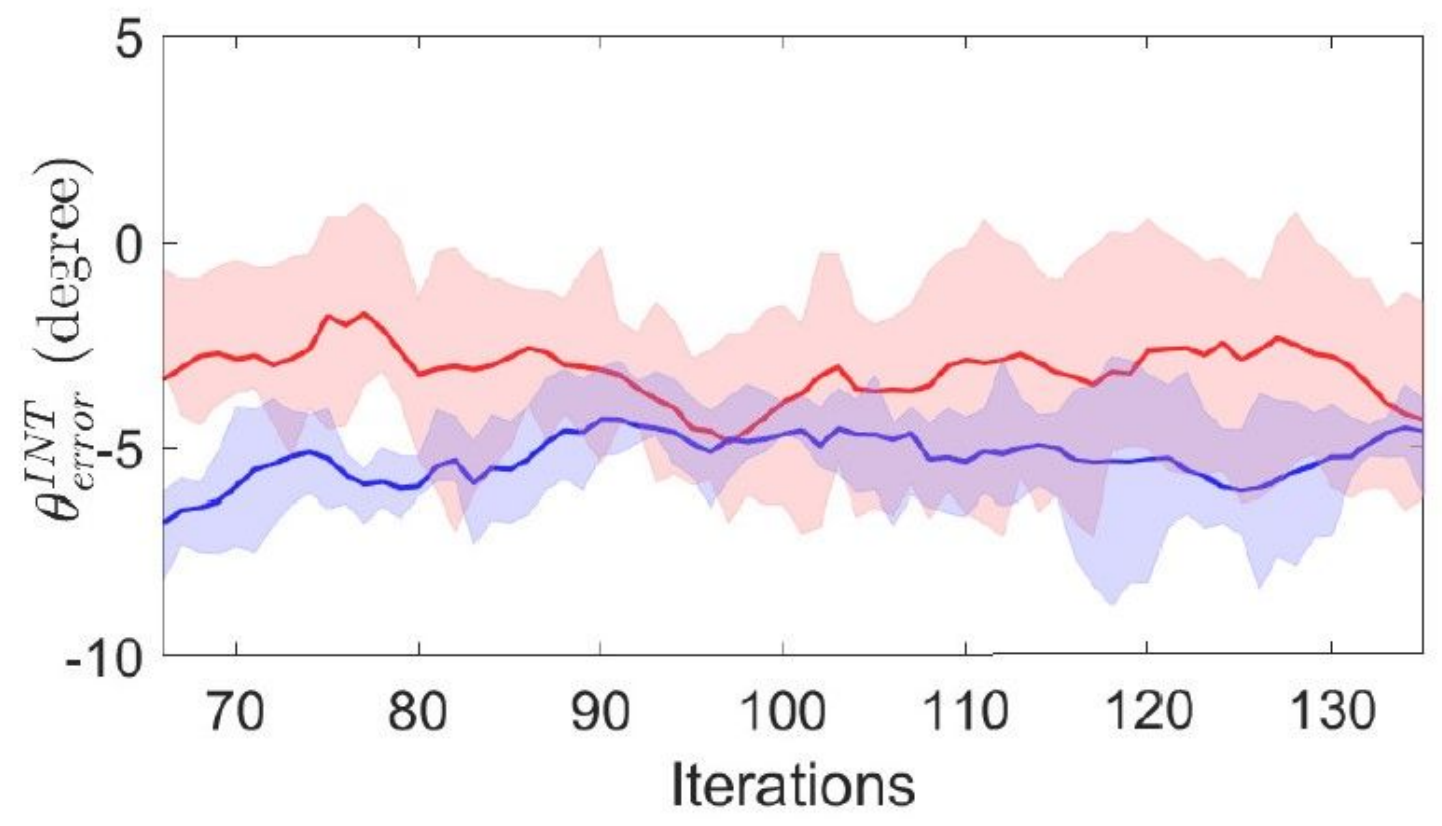

Figure 6 Error over INT phase for the two groups The red and blue solid line shows the mean error in each iteration for all subjects in MGP group and Control group respectively. Shaded areas represent the interquartile range after rejecting outliers (three-sigma rule).

Figure 6 


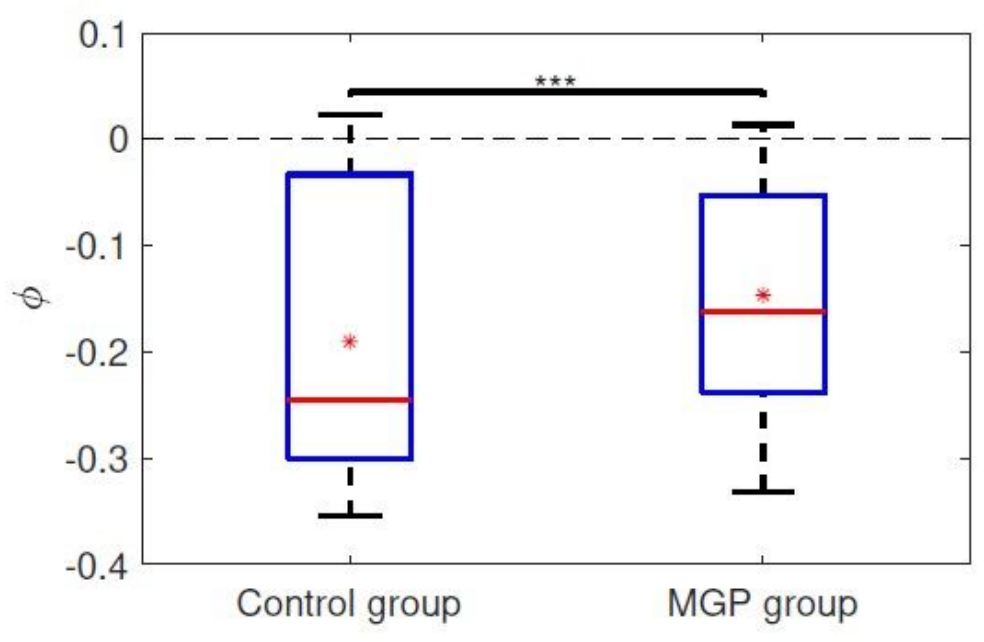

Figure 7 Velocity changes ratio from two groups The box plot shows velocity changes ratio $\phi$ in INT phase for Control group and MGP group. The bottom and top edges of the blue box indicate the 25 th and 75 th percentiles respectively while the red + shows the outliers. Inside the blue box, the red solid line shows the median and the red star shows the mean value of the data set. *: $p<0.05, * *: p<0.01, * * *: p<0.001$.

\section{Figure 7}

\section{Supplementary Files}

This is a list of supplementary files associated with this preprint. Click to download.

- references.bib

- bmcart.cls

- bmcartbiblio.sty

- main.tex

- bmcmathphys.bst 